Int. Journal of Game Theory, Vol. 5, Issue 4, page 173-182. Physica-Verlag, Vienna.

\title{
On a Repeated Game Without a Recursive Structure
}

\author{
By J.-F. Mertens, Louvain ${ }^{1}$ ) and $S$. Zamir, Jerusalem ${ }^{2}$ )
}

\begin{abstract}
The solution is given here for the infinitely repeated two-person zero-sum games of incomplete information characterized by $2 \times 2$ games, with information matrices $\left(\begin{array}{ll}a & b \\ b & b\end{array}\right)$ for the first game and $\left(\begin{array}{ll}b & b \\ b & a\end{array}\right)$ for the second game.
\end{abstract}

\section{Introduction}

Two main classes of repeated two person zero-sum games with incomplete information are solved up to now:

- Games in which the information matrices matrices may depend on the player but not on the state of nature [Mertens and Zamir, Mertens].

- Games in which the information matrices do not depend on the players, may depend on the state of nature, with the additional assumption that each player recalls all prior moves [Kohlberg and Zamir, Kohlberg].

It seems that without those assumptions one loses the recursive structure that made those cases tractable.

Here an example is solved of a game not fulfilling those assumptions. It was mentioned as an open problem some six years ago [Zamir]:

There are two possible states of nature and accordingly two payoff matrices, $A=\left(\begin{array}{ll}a_{11} & a_{12} \\ a_{21} & a_{22}\end{array}\right)$ and $B=\left(\begin{array}{ll}b_{11} & b_{12} \\ b_{21} & b_{22}\end{array}\right)$, the actual payoff matrix (i.e. the actual state of nature) is chosen once and for all by the referee (with probability $p$ for matrix $A$ ), and told to neither player. There are in addition two information matrices $H^{\mathrm{A}}=$ $=\left(\begin{array}{ll}a & b \\ b & b\end{array}\right)$ and $H^{B}=\left(\begin{array}{ll}b & b \\ b & a\end{array}\right), a$ and $b$ being two different letters. After each stage, if $T$ is the true payoff matrix ( $A$ or $B$ ), and the players I and II played their pure strategies $i$ and $j$ respectively, the referee transfers $t_{i j}$ from player II's account to player I's and tell both players the letter $H_{i j}^{T}$. The players get no statement on their accounts before the end of the game. It is crucial here that the moves $i$ and $j$ are not stated explicitly by the referee. However, each player recalls his own move ( $i$ or $j$ ) and all his own previous moves in addition to the information statements $H_{i j}^{T}$ made by the referee up to that stage.

\footnotetext{
1) Prof. Jean-Francois Mertens, Université Catholique de Louvain, Belgium.

$\left.{ }^{2}\right)$ Prof. Shmuel Zamir, The Hebrew University of Jerusalem, Israel.
} 
Notice that as soon as the letter $a$ is announced by the referee, the true matrix is revealed to both players.

The payoff in the infinitely repeated game is thought of as being $\lim _{n \rightarrow \infty} E\left(\frac{1}{n} \sum_{k=1}^{n} t_{i_{k} j_{k}}\right)$, but is not defined due to the possible non existence of the limit. Nevertheless we will show that Min Max (and dually Max Min) of the infinite game exists in a well defined (and rather strong) sense:

Player II has an infinite game strategy that guarantees even in all sufficiently large finite games $E\left(\frac{1}{n} \sum_{k=1}^{n} t_{i_{k} j_{k}}\right)<\operatorname{Min} \operatorname{Max}+\epsilon$; conversely, for every infinite game strategy $\tau$ of player II, player I has an infinite game strategy $\sigma$ such that $\liminf _{n \rightarrow \infty} E\left(\frac{1}{n} \sum_{k=1}^{n} t_{i_{k} j_{k}}\right)>$ $>\operatorname{Min} \operatorname{Max}-\epsilon$.

For a proof of this result let us introduce a few conventions: We may obviously substract from the matrices $A$ and $B$ their values $v(A)$ and $v(B)$ respectively, which will substract from all payoffs the constant $p v(A)+(1-p) v(B)$. Hence we may assume without loss of generality that $v(A)=v(B)=0$. We may multiply $A$ by $p$ and $B$ by $(1-p)$, and consider the payoff to be the sum of the payoffs that would be obtained if $A$ was the true matrix and if $B$ was the true matrix. We will do this in order to simplify slightly notations. Finally $x^{\prime}$ will always stand for $1-x$.

1. We define the following auxiliary game $\bar{\Gamma}$ :

$\tilde{T}$
$\tilde{B}$
$\left(1 \sim_{\epsilon}^{\sim} \sim_{\epsilon}\right) T$
$B_{1}$
$T_{1}$$\left[\begin{array}{ccc}\tilde{L} & \tilde{R} & \left(\beta, \tilde{\beta}^{\prime}\right) \\ b_{11} & a_{12}+b_{12} & \beta b_{11}+\beta^{\prime} b_{12} \\ a_{21}+b_{21} & a_{22} & \beta a_{21}+\beta^{\prime} a_{22} \\ b_{11} & a_{12} & 0 \\ b_{21} & a_{22} & 0 \\ b_{11} & a_{12} & \beta\left(\beta b_{11}+\beta^{\prime} b_{12}\right) \\ b_{21} & a_{22} & \beta^{\prime}\left(\beta a_{21}+\beta^{\prime} a_{22}\right)\end{array}\right]$

Here $\widetilde{L}$ (resp. $\widetilde{R}, \widetilde{T}, \widetilde{B}$ ) stands for the strategy (of player II) of playing always Left (resp. Right, Top, Bottom); $(1 \simeq \epsilon) T$ (resp. $(1 \simeq \epsilon) B$ ) stands for the strategy of playing at every stage independently with probability $(1-\epsilon)$ Top (resp. $B$ ) and with probability $\epsilon$ Bottom (resp. $T) .\left(\beta, \beta^{\prime}\right)$ stands for strategy of playing at each stage and independently with probability $\beta$ Left and with probability $\beta^{\prime}$ Right. Finally $T_{1}$ (resp. $B_{1}$ ) stands for a strategy consisting of playing once $T$ (resp. $B$ ) and all other times $B$ (resp. $T$ ). The entries $\bar{\Gamma}$ can be easily obtained as asymptotic payoffs corresponding to those strategies, using our previous conventions (and thinking of $\beta$ as strictly between 0 and 1 ). 
Denote by $\bar{\nu}$ the value of $\bar{\Gamma}$. If we denote by Max Min $\Gamma$ and Min Max $\Gamma$ the max Min and Min Max value of our original game in the strong sense that we described we shall prove that Min Max $\Gamma=\bar{v}$ and that it may be different from Max Min $\Gamma$. To make these statements rigorous we need still two more definitions:

2. Let us define:

$$
\begin{aligned}
& \bar{v}_{\infty}=\inf \left\{U \mid \exists N \exists \tau \text { s.t. } \forall n \geqslant N \forall \sigma_{n}, \rho_{n}\left(\sigma_{n}, \tau\right) \leqslant U\right\} \\
& \widetilde{v}_{\infty}=\sup \left\{U \mid \forall \tau, \exists \sigma \text { s.t. } \lim _{n \rightarrow \infty} \inf \rho_{n}(\sigma, \tau) \geqslant U\right\}
\end{aligned}
$$

where $\sigma$ (resp. $\tau$ ) stands for a strategy of player I (resp. II) in the infinite game while $\sigma_{n}$ (resp. $\tau_{n}$ ) stands for a strategy of player I (resp. II) in a game consisting of $n$ stages only. $\rho_{n}(\sigma, \tau)$ is the expected average payoff per stage in the first $n$ stages, given $\sigma, \tau$ and $p$; i.e. $\rho_{n}(\sigma, \tau)=E_{\sigma, \tau, p}\left(\frac{1}{n} \sum_{k=1}^{n} t_{i_{k} j_{k}}\right)$, where $T=\left(t_{i j}\right)$ is the true payoff matrix chosen by the referee at the begining of the game.

Loosely speaking, $\bar{v}_{\infty}$ is the lowest value of $\lim \sup \rho_{n}$ that player II can guarantee in the infinite game while $\tilde{v}_{\infty}$ is the highest value of $\lim$ inf $\rho_{n}$ that cannot be guaranteed by player II. Clearly $\tilde{v}_{\infty} \leqslant \bar{v}_{\infty}$. In the next section we will prove that $\bar{v}=\bar{v}_{\infty}=\tilde{v}_{\infty}$, which establishes that $\bar{v}$ is $\operatorname{Min} \operatorname{Max} \Gamma$ in the above explained sense.

\section{Proofs}

For a strategy of player $I$ in $\bar{\Gamma}$ let $\left(\alpha, \alpha^{\prime}\right)$ be the probability distribution induced on $(1 \simeq \epsilon) T$ and $(1 \simeq \epsilon) B$

Lemma 1 . For any $\alpha$ corresponding to any undominated optimal strategy of Player I in $\bar{\Gamma}$ that uses $(1 \simeq \epsilon) T$ or $(1 \stackrel{\sim}{\simeq}) B$ with a positive probability, one of the following holds:

$$
\begin{aligned}
& \alpha a_{12} \geqslant 0 \text { and } \alpha^{\prime} b_{21} \geqslant 0 \\
& \alpha b_{11} \geqslant 0 \text { and } \alpha^{\prime} a_{22} \geqslant 0
\end{aligned}
$$

Proof. Assume that for some optimal $\alpha$ neither of (3.1) and (3.2) holds, so for instance $\alpha b_{11}<0$ (the case $\alpha^{\prime} a_{22}<0$ is completely symmetric). Since $\alpha b_{11}<0$ and $v(B)=0$, we have $b_{21} \geqslant 0$ and thus also $\alpha a_{12}<0$ ( since 3.1) does not hold). Since $\alpha a_{12}<0$ and $v(A)=0$, we have $a_{22} \geqslant 0$. But this implies that the strategy $(1 \simeq \epsilon) B$ of player I strictly dominates his strategy $(1 \simeq \epsilon) T$ in $\bar{\Gamma}$, and thus that $\alpha=0$ which contradicts the assumption $\alpha b_{11}<0$. 
Theorem 1. $\tilde{v}_{\infty} \geqslant \bar{v}$

Proof. Consider an arbitrary strategy $\tau$ of player II and an arbitrary $\epsilon(0<\epsilon<1)$. $\tau$ may be considered as a probability measure $P$ on the space $\Omega$ of all sequences of $L$ (left) and $R$ (right) with the understanding that as soon as the true matrix is revealed, player II switches to his optimal strategy in that matrix.

Let $p_{1}=P(\tilde{L}), p_{2}=P(\widetilde{R}), p_{3}=P(\Omega \backslash\{\tilde{L}, \widetilde{R}\})=1-p_{1}-p_{2}$. Let $\Omega_{\infty}$ denote the subset of $\Omega$ consisting of sequences with infinitely many $L$ and infinitely many $R$. Let $\left\{L_{i}\right\}$ denote the subset of $\Omega$ with a finite non-zero number of $L$ in the sequence, and similarly $\left\{R_{i}\right\}$ is the subset of those sequences with a finite non-zero number of $R$. We shall refer to these finitely many $L$ or $R$ as the exceptional moves.

Define $N_{1}$ by:

Prob. [player II has not played all his exceptional moves before $\left.N_{1} \mid\left\{L_{i}\right\} \cup\left\{R_{i}\right\}\right]<\epsilon$ and $N_{2}$ by:

Prob. \{number of $L$ and $R$ in the intervall $] N_{1}, N_{2}$ [ are both at least

$\left.\frac{\ln \epsilon}{\ln (1-\epsilon)} \mid \Omega_{\infty}\right\}>1-\epsilon$

with the understanding that whenever the conditioning set has zero probability, the corresponding integer takes its least possible value $\left(1\right.$ or $\left.N_{1}+1\right)$.

It follows from the definitions that even if player I plays $(1-\epsilon) B$ in $N_{1}, N_{2}$ [, matrix $A$ (if it is the true matrix) will be revealed with probability greater than $1-2 \epsilon$, given $\Omega_{\infty}$, and also that:

Prob. [Both $L$ and $R$ appear before $\left.N_{2} \mid \Omega \backslash(\{\tilde{L}\} \cup\{\tilde{R}\})\right]>1-\epsilon$

Let $\left(q_{1}, q_{2}, \alpha q_{3}, \alpha^{\prime} q_{3}, q_{4}, q_{5}\right)$ be an undominated optimal strategy of player $I$ in

$\bar{\Gamma}$. For any $k>N_{2}$, let $\sigma_{k}$ be the following strategy of player I:

- with probability $q_{1}$, play $\tilde{T}$

- with probability $q_{2}$, play $\widetilde{B}$

- with probability $q_{3}$, choose $H$ with probability $\alpha$ and $S$ with probability $\alpha^{\prime}$ and play:

- if (3.1) holds: if $H: \widetilde{B}$ up to $N_{1}$ and $(1 \simeq \epsilon) T$ after $N_{1}$.

if $\breve{S}: \widetilde{T}$ up to $N_{1}$ and $(1 \simeq \dot{\sim}) B$ after $N_{1}$.

- if (3.2) holfs: if $H: \widetilde{T}$ up to $N_{1}$ and $(1 \simeq \epsilon) T$ after $N_{1}$. if $S: \widetilde{B}$ up to $N_{1}$ and $(1 \simeq \epsilon) B$ after $N_{1}$.

- with probability $q_{4}$, play a strategy $B_{1}$, with the time of playing $B$ chosen independently of all other choices and uniformly in $[1, k]$.

- with probability $q_{5}$, play a strategy $T_{1}$, with the time of playing $T$ chosen independently of all other choices and uniformly in $[1, k]$.

We have for all $n>k$,

$$
1 / n \leqslant N_{1} / n \leqslant N_{2} / n \leqslant k / n ; 1 / n \epsilon \leqslant k / n, 1 / k \leqslant \epsilon .
$$

Let $f_{n}=(1 / n) \cdot($ number of $L$ up to time $n)$. 
Let $M=\max \left[\max _{i, j} a_{i j}-\min _{i, j} a_{i j} ; \max _{i, j} b_{i j}-\min _{i, j} b_{i j}\right]$,

and let $O(\epsilon)$ stand for any quantity $x$ such that $|x| \leqslant M \epsilon$

similarly $0(1 / k)$ stands for any $y$ such that $|y| \leqslant M / k$, etc.

Denote by $\rho_{n}\left(\sigma_{k}, \tau\right)$ the average payoff per stage resulting from strategies $\sigma_{k}$ and $\tau$, we have that $\rho_{n}\left(\sigma_{k}, \tau\right)$ is the expectation of:

$$
\begin{aligned}
& q_{1}\left[p_{1}\left(b_{11}+0(1 / n)\right)+p_{2}\left(a_{12}+b_{12}\right)+p_{3}\left(f_{n} b_{11}+f_{n}^{\prime} b_{12}\right)+0\left(N_{2} / n\right)+0(\epsilon)\right] \\
& +q_{2}\left[p_{1}\left(a_{21}+b_{21}\right)+p_{2}\left(a_{22}+0(1 / n)\right)+p_{3}\left(f_{n} a_{21}+f_{n}^{\prime} a_{22}+0\left(N_{2} / n\right)+0(\epsilon)\right)\right] \\
& +q_{3}\left[p _ { 1 } \left\{\alpha b_{11}+\alpha^{\prime} b_{21}+0\left(N_{1} / n\right)+20(\epsilon)+(0(1 / n \epsilon) \text { if (3.2) holds) }\}+\right.\right. \\
& p_{2}\left\{\alpha a_{12}+\alpha^{\prime} a_{22}+0\left(N_{1} / n\right)+20(\epsilon)+(0(1 / n \epsilon) \text { if (3.2) holds })\right\}+ \\
& P\left(\Omega_{\infty}\right)\left(40(\epsilon)+0\left(N_{2} / n\right)\right)+ \\
& \left\{\begin{array}{l}
\text { if }(3.1) \text { holds: } \\
P\left(\left\{L_{i}\right\}\right)\left(\alpha a_{12}+30(\epsilon)+0\left(N_{1} / n\right)\right)+p^{\prime}\left(\left\{R_{i}\right\}\right)\left(\alpha^{\prime} b_{21}+30(\epsilon)+0\left(N_{1} / n\right)\right) \\
\text { if }(3.2) \text { holds: } \\
\begin{array}{rl}
P\left(\left\{L_{i}\right\}\right)\left(\alpha^{\prime} a_{22}+30(\epsilon)+0\left(N_{1} / n\right)+0(1 / n \epsilon)\right) & +P\left(\left\{R_{i}\right\}\right)\left(\alpha b_{11}\right. \\
& \left.+30(\epsilon)+0\left(N_{1} / n\right)+0(1 / n \epsilon)\right)
\end{array}
\end{array}\right. \\
& +q_{4}\left[p_{1}\left(b_{11}+0(1 / n)\right)+p_{2}\left(a_{12}+0(k / n)\right)+p_{3}\left\{f_{k}\left(f_{n} b_{11}+f_{n}^{\prime} b_{12}\right)+0(1 / k)\right.\right. \\
& +0(\epsilon)+0(k / n)\}] \\
& +q_{5}\left[p_{1}\left(b_{21}+0(k / n)\right)+p_{2}\left(a_{22}+0(1 / n)\right)+p_{3}\left\{f_{k}^{\prime}\left(f_{n} a_{21}+f_{n}^{\prime} a_{22}\right)+0(1 / k)\right.\right. \\
& +0(\epsilon)+0(k / n)\}]
\end{aligned}
$$

Using relations (3.3) and Lemma 1 we get that for all $n>k$ :

$$
\rho_{n}\left(\sigma_{k}, \tau\right) \geqslant E(H(k, n, \tau, \omega))-4 M \epsilon-0(k / n)
$$

where

$$
H(k, n, \tau, \omega)=\left[\begin{array}{c}
q_{1} \\
q_{2} \\
\alpha q_{3} \\
\alpha^{\prime} q_{3} \\
q_{4} \\
q_{5}
\end{array}\right] \cdot\left[\begin{array}{cccc}
b_{11} & a_{12}+b_{12} & b_{11} & b_{12} \\
b_{21}+b_{21} & a_{22} & a_{21} & a_{22} \\
b_{11} & a_{12} & 0 & 0 \\
b_{21} & a_{22} & 0 & 0 \\
b_{11} & a_{12} & f_{k} b_{11} & f_{k} b_{12} \\
b_{21} & a_{22} & f_{k}^{\prime} a_{21} & f_{k}^{\prime} a_{22}
\end{array}\right]\left[\begin{array}{l}
p_{1} \\
p_{2} \\
f_{n} p_{3} \\
f_{n}^{\prime} p_{3}
\end{array}\right]
$$


Denote $E(H(k, n, \tau, \omega))$ by $\phi\left(f_{k}, f_{n}\right)$. The function $\phi: L_{\infty} \times L_{\infty} \rightarrow \underset{\sim}{R}$ is weakly continuous and affine in each variable separately on $L_{\infty}$ endowed with the weak topo$\operatorname{logy} \sigma\left(L_{\infty}, L_{1}\right)$.

Let $C=$ closed convex hull of $\left\{f_{i} \mid i>N_{2}\right\}$ in $L_{\infty}-\sigma\left(L_{\infty}, L_{1}\right)$, and consider $\phi$ on $C \times C$ : We have $\phi(f, f) \geqslant \bar{v} \forall f \in C$, indeed for $f_{k}=f_{n}=f, H(k, n, \tau, \omega) \geqslant \bar{v}$ holds for each value of $\omega$ since $\left(q_{1}, q_{2}, \alpha q_{3}, \alpha^{\prime} q_{3}, q_{4}, q_{5}\right)$ is an optimal strategy of player $I$ in $\bar{\Gamma}$. In addition $C$ is compact and convex for $\sigma\left(L_{\infty}, L_{1}\right)$ and $\phi$ is affine and continuous in each variable separately on $C$. It follows that $\phi$ has a saddle point, hence:

$$
\exists g \in C \text { s.t. } \forall f \in C: \phi(g, f) \geqslant \inf _{f} \sup _{u} \phi(u, f) \geqslant \inf _{f} \phi(f, f) \geqslant \bar{v}
$$

Now $g$ is also in the closure of the convex hull of $\left\{f_{i} \mid i>N_{2}\right\}$ when $L_{\infty}$ is endowed with the Mackey topology $\tau\left(L_{\infty}, L_{1}\right)$ - due to the convexity of the set - (this is a well known result that follows from the Hahn-Banach theorem). Since on bounded sets of $L_{\infty}$ the Mackey topology $\tau\left(L_{\infty}, L_{1}\right)$ coincides with the topology of convergence in probability, it follows that there exist $\lambda_{i}\left(1 \leqslant i \leqslant l, \lambda_{i} \geqslant 0, \sum_{i=1}^{l} \lambda_{i}=1\right)$ and $k_{i}\left(1 \leqslant i \leqslant l, k_{i}>N_{2}\right)$ such that:

$$
P\left(\left|\sum_{i=1}^{l} \lambda_{i} f_{k_{i}}-g\right| \geqslant \epsilon\right)<\epsilon
$$

Let now $\sigma_{\epsilon, \tau}$ be the strategy of player I consisting of choosing at the start of the game a number $i(1 \leqslant i \leqslant l)$ with probability $\lambda_{i}$, and thereafter using his strategy $\sigma_{k_{i}}$. Let also $K_{M}=\max \left\{k_{i} \mid 1 \leqslant i \leqslant l\right\}$ then we have:

$$
\rho_{n}\left(\sigma_{\epsilon, \tau}, \tau\right) \geqslant \phi\left(g, f_{n}\right)-6 M \epsilon-0\left(K_{M} / n\right) \text { for all } n>K_{M}
$$

Thus: $\forall \tau$, strategy of player II, $\forall \epsilon, 0<\epsilon<1, \exists \sigma_{\epsilon, \tau}$, strategy of player I, such that:

$$
\lim _{n \rightarrow \infty} \inf \rho_{n}\left(\sigma_{\epsilon, \tau}, \tau\right) \geqslant \inf _{f \in C} \phi(g, f)-6 M \epsilon \geqslant \bar{v}-6 M \epsilon
$$

This completes the proof of Theorem 1.

Lemma 2. Player II has an optimal strategy in $\bar{\Gamma}$ using only a single value of $\beta$.

Proof. A priori player Il's optimal strategy in $\bar{\Gamma}$ consists of a probability vector $\left(p_{1}, p_{2}, p_{3}\right)$ together with a probability measure $\mu$ on $[0,1]$ to choose $\beta$. We want to show that player II has an optimal strategy in which $\mu$ 's support is a single point in $[0,1]$.

L 1) If $b_{11} \geqslant b_{12}$ and $a_{22} \geqslant a_{21}$ the result follows from the convexity in $\beta$ of the payoff function. 
L 2) Otherwise we have either $b_{11}<b_{12}$ or $a_{22}<a_{21}$, by symmetry we may assume that $a_{22}<a_{21}$. Since $v(A)=0$ it follows that $a_{22} \leqslant 0$.

L 2.1) If in addition $b_{11} \leqslant b_{12}$ the payoff function is concave in $\beta$ and thus $\mu$ is dominated by the probability on $\{0,1\}$ that has the same mean. So without loss of generality we may assume that in this case $\mu(] 0,1[)=0$. We get thus for $\bar{\Gamma}$ a $6 \times 4$ matrix with $\widetilde{L}, \widetilde{R, \beta}=1$ and $\beta=0$ as pure strategies for player II. The other strategies are eliminated by domination. In addition $\nu(B)=0$ implies $b_{11} \leqslant 0$, and thus we conclude that rows $B_{1}$, and $T_{1}$ are dominated by $(1 \simeq \epsilon) T$ and $(1 \simeq \epsilon) B$ respectively. If either $b_{21} \leqslant 0$ or $a_{12} \leqslant 0$, one of the rows $\beta=1$ or $\beta=0$ is dominated by $\widetilde{L}$ or $\widetilde{R}$ respective. ly and the result follows. If either $a_{21} \leqslant 0$ or $b_{12} \leqslant 0$, say $a_{21} \leqslant 0$ then first $\widetilde{B}$ is dominated by $(1 \sim \epsilon) B$ and then $\beta=0$ is dominated by $\beta=1$; the result follows again. Thus we may assume that $\operatorname{Min}\left(a_{12}, a_{21}, b_{12}, b_{21}\right)>0$, it follows then from $v(A)=v(B)=0$ that $b_{11}<0, a_{22}<0$.

$$
\text { Let } \begin{aligned}
R=\left(\begin{array}{ll}
b_{11} & b_{12} \\
a_{21} & a_{22}
\end{array}\right), & C=\left(\begin{array}{ll}
b_{11} & a_{12} \\
b_{21} & a_{22}
\end{array}\right) \\
r=\operatorname{Val}(R), & c=\operatorname{Val}(C)
\end{aligned}
$$

L 2.1.1) If $r \leqslant 0$ and if we denote by $\left(\beta, \beta^{\prime}\right)$ the relative weights of the columns $\beta=1$ and $\beta=0$, then there exists an optimal $\beta$ for which $\beta b_{11}+\beta^{\prime} b_{12} \leqslant 0$ and $\beta a_{21}+\beta^{\prime} a_{22} \leqslant 0$ (if $c<0$, the required $\beta$ is the relative weight of the last two columns in the equalizing strategy of player II, if $c \geqslant 0$ the value of the game is 0 and an optimal strategy of player II is $\left(0,0, \beta, \beta^{\prime}\right)$ where $\beta$ is optimal in $R$ and hence satisfies the required inequalities). It follows that if in that optimal strategy, player II would replace the columns $\beta=1$ and $\beta=0$ by i.i.d. $(\beta, \beta)$, rows $B_{1}$ and $T_{1}$ would still be dominated by $(1 \sim \epsilon) T$ and $(1 \sim \epsilon) B$ respectively and hence player II has in this case an optimal strategy using a single $\beta$.

L 2.1.2) If $r>0$, the optimal mixture of the columns $\beta=1$ and $\beta=0$ is $\left(\beta, \beta^{\prime}\right), \beta$ being optimal in $R$ and hence $\beta b_{11}+\beta^{\prime} b_{12}>0$ and $\beta a_{21}+\beta^{\prime} a_{22}>0$. It follows again that replacing the last two columns by i.i.d. $\left(\beta, \beta^{\prime}\right)$, rows $B_{1}$ and $T_{1}$ remain dominated, this time by $\widetilde{T}$ and $\widetilde{B}$ respectively, providing again a single $\beta$ optimal strategy for player II.

L 2.2) We are thus left with the case:

$$
a_{22}<a_{21}, a_{22} \leqslant 0, b_{12}<b_{11}, b_{12} \leqslant 0 \text {. }
$$

Consider player II's optimal strategy in the game $\bar{\Gamma}$ without the rows $B_{1}$ and $T_{3}$; it obviously implies $\beta=0$. For this $\beta, B_{1}$ is dominated by $(1 \simeq \epsilon) T$ and $T_{1}$ by $(1 \simeq \epsilon) B$ and thus this single $\beta$ strategy is also optimal in $\vec{\Gamma}$. This completes the proof of Lemma 2 .

Notice that the strategies $\beta=1$ and $\beta=0$ in $\bar{\Gamma}$ should be interpreted as playing i.i.d. $(1 \simeq \epsilon, \epsilon)$ and $(\epsilon, \tilde{1}-\epsilon)$ respectively. Thus in the single $\beta$ optimal strategy for player II established in Lemma 2 we may assume $0<\beta<1$. 
Theorem 2. $\bar{v}_{\infty} \leqslant \bar{v}$.

Proof. We will show that whenever player II plays in $\Gamma$ one of his strategies $\tau$ in $\bar{\Gamma}$, consisting of a mixture of $\tilde{L}, \widetilde{R}$ and one $\left(\beta, \tilde{\beta}^{\prime}\right)-$ with $0<\beta<1-$ any pure strategy of player I yields in $\Gamma_{n}$ a payoff dominated up to terms $0(1 / n)$ by a convex combination of rows of $\bar{\Gamma}$. Since by Lemma 2 player II can guarantee $\bar{v}-$ up to $\epsilon-$ by such mixtures $\tau$ against rows of $\vec{\Gamma}$, the result will then follow.

If the pure strategy of player I is $\widetilde{T}$ or $\widetilde{B}$ then it is already a row of $\bar{\Gamma}$. Take any other pure strategy that begins say with $T$ (for strategies starting with $B$ the discussion is completely dual). Let $\omega_{i}=1$ if $T$ occurs at time $i$ in the strategy and $\omega_{i}=0$

otherwise. Let $f_{n}=\frac{1}{n} \sum_{i=1}^{n} \omega_{i}$, and $\omega_{t+1}$ be the first zero in the sequence $\left\{\omega_{i}\right\}$. Let $y=t / n ;$ we have $1 / n \leqslant y \leqslant f_{n} \leqslant(n-1) / n$.

Let $D=\beta b_{11}+\beta^{\prime} b_{12}, \quad G=\beta a_{21}+\beta^{\prime} a_{22}$,

$$
\begin{aligned}
& X=\frac{1}{n}\left[\omega_{1}^{\prime}+\omega_{2}^{\prime}\left(1-\beta \omega_{1}\right)+\ldots+\omega_{n}^{\prime}\left(1-\beta \omega_{1}\right) \ldots\left(1-\beta \omega_{n-1}\right)\right] \\
& Y=\frac{1}{n}\left[\omega_{1}+\omega_{2}\left(1-\beta^{\prime} \omega_{1}^{\prime}\right)+\ldots+\omega_{n}\left(1-\beta^{\prime} \omega_{1}^{\prime}\right) \ldots\left(1-\beta^{\prime} \omega_{n-1}^{\prime}\right)\right] .
\end{aligned}
$$

We have $f_{n}^{\prime} \beta^{\prime n f_{n}} \leqslant X \leqslant f_{n}^{\prime} \beta^{\prime n y}$ and

$$
y+\left(f_{n}-y\right) \beta^{n f^{\prime}} \leqslant Y \leqslant y+\left(f_{n}-y\right) \beta, \text { neglecting terms } 0(1 / n) .
$$

The strategy of player I obtains, up to $O(1 / n)$; against $\widetilde{L}: f_{n} b_{11}+f_{n}^{\prime} b_{21}$; against $\widetilde{R}: y b_{12}+f_{n} a_{12}+f_{n}^{\prime} a_{22}$ and against $\left(\beta, \sim^{\prime}\right): G X+D Y$. Majorizing this last term according to the sign of $G$ and $D$ we obtain (writing $f$ for $f_{n}$ );

\begin{tabular}{l|c|c} 
against & $\tilde{L}$ & $\tilde{R}$ \\
\hline a payoff $\leqslant$ & $f b_{11}+f^{\prime} b_{21}$ & $y b_{12}+f a_{12}+f^{\prime} a_{22}$
\end{tabular}

\begin{tabular}{c|l|l|l|l} 
against & \multicolumn{4}{|c}{$\left(\beta^{-} \beta^{\prime}\right)$ with: } \\
\hline & $G \geqslant 0 . D \geqslant 0$ & $G<0 . D \geqslant 0$ & $G \geqslant 0 . D<0$ & $G<0 . D<0$ \\
\hline a payoff $\leqslant$ & $G f^{\prime} \beta^{\prime n y}+D\left(\beta f^{+}+\beta^{\prime} y\right)$ & $G f^{\prime} \beta^{\prime m f}+D\left(\beta f+\beta^{\prime} y\right)$ & $G f^{\prime} \beta^{\prime n y}+D\left(y+(f-y) \beta^{n f^{\prime}}\right)$ & $G f^{\prime} \beta^{\prime} n f_{+D\left(y+(f-y) \beta^{n f^{\prime}}\right)}$
\end{tabular}

Since all terms are convex in $y$, we may replace $y$ by its extreme values $1 / n$ and $f$. Neglecting terms $0(1 / n)$ one gets thus:

\begin{tabular}{c|c|c|l|l|l|l} 
& \multirow{2}{*}{$\tilde{L}$} & $\tilde{R}$ & \multicolumn{5}{|c}{$\left(\beta^{\prime} \beta^{\prime}\right)$ with: } \\
\cline { 4 - 8 } & & $G \geqslant 0 . D \geqslant 0$ & $G<0 . D \geqslant 0$ & $G \geqslant 0 . D<0$ & $G<0 . D<0$ \\
\hline$y=1 / n$ & $f b_{11}+f^{\prime} b_{21}$ & $f a_{12}+f^{\prime} a_{22}$ & $G f^{\prime} \beta^{\prime}+D f \beta$ & $G f^{\prime} \beta^{\prime n f}+D f \beta$ & $G f^{\prime} \beta^{\prime}+D f^{n f^{\prime}}$ & $G f^{\prime} \beta^{\prime n f}+D f \beta^{n f^{\prime}}$ \\
\hline$y=f$ & $f b_{11}+f^{\prime} b_{21}$ & $f\left(a_{12}+b_{12}\right)+f^{\prime} a_{22}$ & $G f^{\prime} \beta^{\prime n f}+D f$ & $G f^{\prime} \beta^{\prime n f}+D f$ & $G f^{\prime} \beta^{\prime n f}+D f$ & $G f^{\prime} \beta^{\prime n f}+D f$ \\
\hline
\end{tabular}


$f \beta^{n f^{\prime}}$ and $f^{\prime} \beta^{\prime n f}$ are convex. When their coefficients are negative let us majorize them by zero. All functions get then linear or convex in $f$, so we may replace $f$ by its extreme values $1 / n$ and $(1-1 / n)$. Neglecting terms $0(1 / n)$ one obtains:

\begin{tabular}{l|c|c|c|c|c|c} 
& & & \multicolumn{5}{|c}{$\left(\beta . \beta^{\prime}\right)$ with: } \\
\cline { 4 - 7 } & $\tilde{L}$ & $\widetilde{R}$ & $G \geqslant 0 . D \geqslant 0$ & $G<0, D \geqslant 0$ & $G \geqslant 0 . D<0$ & $G<0 . D<0$ \\
\hline$y=1 / n, f=1-1 / n$ & $b_{11}$ & $a_{12}$ & $D \beta$ & $D \beta$ & 0 & 0 \\
\hline$y=f=1 / n$ & $b_{21}$ & $a_{22}$ & $G \beta$ & 0 & $G \beta^{\prime}$ & 0 \\
\hline$y=f=1-1 / n$ & $b_{11}$ & $a_{12}+b_{12}$ & $D$ & $D$ & $D$ & $D$ \\
\hline
\end{tabular}

We conclude that player I's strategy is dominated by the mixture of three similar strategies with $(y=1 / n, f=1-1 / n),(y=f=1 / n)$ and $(y=f=1-1 / n)$, the weights being $f-y, f^{\prime}$ and $y$ respectively. But this mixture is dominated by the convex combination with the same weights of the following rows of $\bar{\Gamma}$ :

\begin{tabular}{c|c|c|c|c} 
case & $G \geqslant 0, D \geqslant 0$ & $G<0, D \geqslant 0$ & $G \geqslant 0, D<0$ & $G<0, D<0$ \\
\hline $\begin{array}{c}\text { weights } \\
f^{\prime}-y\end{array}$ & $B_{1}$ & $B_{1}$ & $(1 \simeq \epsilon) T$ & $(1 \simeq \epsilon) T$ \\
\hline$f^{\prime}$ & $T_{1}$ & $(1 \simeq \epsilon) B$ & $T_{1}$ & $(1 \simeq \epsilon) B$ \\
\hline$y$ & $\widetilde{T}$ & $\widetilde{T}$ & $\widetilde{T}$ & $\tilde{T}$ \\
\hline
\end{tabular}

This completes the proof of Theorem 2 .

\section{Conclusions}

(i) $\bar{v}_{\infty}=\tilde{v}_{\infty}=\bar{v}=\operatorname{Min} \operatorname{Max} \Gamma$

(ii) Player II has an " $\epsilon-$ MinMax" strategy of the type: With probability $p_{1}$ play always $L$, with probability $p_{2}$ play always $R$ and with probability $1-p_{1}-p_{2}$ play always i.i.d. with probability $\beta, L$ and with probability $\beta^{\prime}, R$.

(iii) This strategy also guarantees that in any finite sufficiently long game the payoff is less than $\bar{v}+\epsilon$.

(iv) Dual results hold for player I.

(v) Analysis of the game $\bar{\Gamma}$ and its' dual $\Gamma$ shows that the only cases where there is no value (i.e. $\bar{v}>v$ ) are: $\left\{c \vee r<0\right.$ and $a_{21}>0$ and either $a_{12} \wedge b_{12}>0$ or $\left.a_{12}\left(a_{21}-a_{22}\right)+b_{21} a_{22}<0\right\}$

and its symmetries obtained by either permuting the games

$\left(a_{i j} \leftrightarrow b_{i^{\prime} j^{\prime}}\right.$ where $1^{\prime}=2$ and $\left.2^{\prime}=1\right)$ or permuting the players $\left(a_{i j} \leftrightarrow-\alpha_{j i}\right.$, $b_{i j} \leftrightarrow-b_{i i}$ ) or both. 
An example of a game without value is the following:

$$
A=\left(\begin{array}{rr}
-1 & 2 \\
2 & -4
\end{array}\right), \quad B=\left(\begin{array}{rr}
-4 & 2 \\
2 & -1
\end{array}\right)
$$

Optimal strategies in $\bar{\Gamma}$ are: For player $\mathrm{I} ;(1 / 4,1 / 4,1 / 4,1 / 4,0,0)$ and for player II;

$(1 / 4,1 / 4,1 / 2,(1 / 2,1 / 2))$ giving $\bar{v}=-1 / 2$. Optimal strategies in $\Gamma$ are: For player $\mathrm{I}$ : $(1 / 6,1 / 6,2 / 3 .(1 / 2,1 / 2))$ and for player II; $(1 / 6,1 / 6,0,0,1 / 3,1 / 3)$ giving $v=-2 / 3$.

\section{References}

Kohlberg, E. : Repeated Games with Absorbing States, Annals of Statistics 2, 1974, 724-738.

Kohlberg, E., and S. Zomir: Repeated Games of Incomplete Information: The Symmetric Case, Annals of Statistics. 2 (5), 1974, 1040-1041.

Mertens, J.F.: The Value of Two-Person Zero-Sum Repeated Games, The Extensive Case. International Journal of Game Theory 1 (4), 1971/72, 217-227.

Mertens, J.F., and S. Zamir: The Value of Two-Person Zero-Sum Repeated Games with Lack of Information on Both Sides. International Journal of Game Theory 1, 1971, 39-64.

Zamir, S. : Repeated Games with Incomplete Information, Ph.D. Thesis, Department of Mathematics, The Hebrew University, Jerusalem, Isreal 1970.

Received October, 1974

(revised version February, 1977) 